\title{
Interface Lessons for Fully and Semi-Autonomous Mobile Robots
}

\author{
Aaron Steinfeld \\ The Robotics Institute \\ Carnegie Mellon University \\ Pittsburgh, Pennsylvania \\ steinfeld@cmu.edu
}

\begin{abstract}
Experts from the Robotics Institute were individually interviewed for their insight on interface lessons for fully and semi-autonomous mobile robots. Information was collected on four main themes: challenges, things that seem to work well, things that do not work well, and interface wisdom. The comments were then condensed and pooled into seven high-level categories: safety, remote awareness, control, command inputs, status and state, recovery, and interface design. Classification of expert comments was relatively straightforward in that many interviewees identified consistent material. This suggests that those producing interfaces for fully and semi-autonomous mobile robots should, at the minimum, ensure that they have addressed these broad topics.
\end{abstract}

Keywords-component; human-robot interaction, mobile robots, interfaces, autonomous, semi-autonomous

\section{INTRODUCTION}

While robotics itself is no longer a nascent field, the subdomain of human interaction with autonomous mobile robots has only recently begun to establish a niche in the research and application community. As such, there has been little documentation of best practices, tarpits, and nuances in this realm. This effort was undertaken to mine the vast experience within the Robotics Institute (RI) on developing and operating autonomous and semi-autonomous mobile robots.

Fortunately, there have been good foundations developed for human interaction with mobile robots and system autonomy. Extensive research on dialog driven human-robot collaboration has shown that robot operation can benefit from requesting help from human operators [1, 2, 3]. A follow-up study on the question and answer interaction method revealed that dialog use was a powerful tool for maintaining system awareness and communicating encountered problems [3]. However, the authors found that this method was tedious for expert users. They also remarked that for collaborative control it might be acceptable to compress the dialog to commands from the human and questions from the robot. This work has also led to recommendations for multiple command generation modes [2] in order to support different operator information needs and tasks.

Potential interface pitfalls when designing interfaces for mobile systems have also been identified. Padmos and van Erp [4] found that drivers of manned ground vehicles could perform moderately well on lateral tasks when driving using a camera view. However a later study comparing headmounted displays with periscopes in an armored vehicle resulted in an alarmingly high incidence of motion sickness [5]. Anecdotal evidence from within RI indicates that teleoperated unmanned robot systems have produced similar operator discomfort even though the operator was not being exposed to physical motion. As a result, basic teleoperation of robots with high lateral and vertical acceleration, due to either the terrain or means of mobility, may be greatly aided by human-robot systems that reduce the need for continuous manual control. These same methods may also reduce errors in real-time manual teleoperation [6] and weaknesses under communication constraints [7]. Readers interested in a detailed examination of vehicle teleoperation interfaces should review [8] and [9].

Research on humans and autonomy has shown that it is not advisable to define the operator's role as a byproduct of how the automation is implemented [10]. Exposure time does matter as past use of automated systems increases likelihood of continued use, even when operators thought they might be able to perform the task better than the automated component [11]. Research on other intelligent systems has produced comparable findings where operators with more exposure time reported higher favorable opinions [12]. Lee and Moray [11] referred to this tendency as "inertia" and recommended designing systems so that reallocation of control was simple. They also suggested that new operators should be encouraged to use automation so that their inherent bias towards manual control would be alleviated.

Safety has already been identified as a major concern with autonomous stationary robots. Fatalities with preprogrammed industrial robots can and do occur as a direct result of human error [13]. Unpredictable robot behavior, especially when developing prototypes, increases the potential for dire consequences. This is especially true in cases where human bystanders lack an interface describing the robot's goals and planned motion. As such, the primary role of the operator and colleagues in the vicinity of the robot is to prevent unsafe scenarios. Related research on mobile robot faults suggests that it is important to try to warn 
operators of irregular robot behavior changes before an unrecoverable catastrophic fault occurs [14].

\section{METHODS}

Experts with extensive autonomous or semi-autonomous mobile robot interface experience within RI were identified and contacted with a request to be interviewed. The six interviews were conducted independently and, with the exception of one conducted via e-mail, were in person and lasted about an hour each. When possible, the expert was interviewed in their own lab or office so that examples would be available. Interviews were kept relatively flexible and only redirected in order to collect material on four main themes: challenges, things that seem to work well, things that do not work well, and interface wisdom.

Following the interview process, the comments were stripped of identifying information and classified into common categories. Comments in each category were then condensed into pooled lessons.

\section{FINDINGS}

Expert comments were pooled into seven categories identified during compilation: safety, remote awareness, control, command inputs, status and state, recovery, and interface design. In most cases, the groupings were rather clear as almost every expert volunteered material for each category.

\section{Safety}

As expected, most of the experts voluntarily indicated that safety is paramount - the vehicle should fail into a safe state. The important detail is that a "safe state" corresponds to a safe state for bystanders as opposed to a safe state for just the robot. It was recommended that emergency stop controls and functionality should be present at both the robot and operator locations. It is not uncommon to see large emergency stop buttons on multiple sides of the more physically dangerous robots at RI.

A related insight was the suggestion that when there are multiple operators, one should consider giving veto power to the operator with a direct line of sight of the robot. Communications latencies, perceptual difficulties (e.g., low field of view video), and reduced situational awareness may prevent a remote operator from making fast decisions.

One expert very clearly identified the calibration and start-up states as requiring critical attention. He recommended that dangerous actions should not be permitted to occur during these states without human authorization or removal of lockouts. This is a valid suggestion as most end users may not expect dangerous robot behavior during these periods. Automobile manufacturers have encountered similar problems with "sudden unintended acceleration" where cars would jump from park or ignition when brake and shift interlocks were not present [15].

\section{Remote Awareness}

The experts were very clear that the interface should provide displays that help the user to understand the remote environment and to maintain situational awareness. At the basic level, it was stated that both video and map views have a place in the interface, it is not a requirement for both to be visible at the same time.

All of the experts described interfaces that provided multiple channels of data at the same time as primary video or map windows (e.g., speed, position, tilt angle, etc). The use of supplementary information in close proximity to video or maps is not surprising given a general bias towards engineering displays and similar information presentation techniques in automobiles and video games.

Robots that provided a forward video scene, even at low resolutions, are ripe for mimicking traditional automotive representations. One expert commented that they had great success utilizing a dashboard layout on the bottom of the screen to represent key information. This method was reported as providing a "fair degree of situational awareness" for even novice users - "people immediately understood what they were looking at." Utilizing the forward sector of $360^{\circ}$ or panoramic cameras in this manner worked well too.

There were also specific comments regarding fused sensor displays (e.g., imagery overlay similar to a head-up display). These were reported as helping situational awareness but prone to pitfalls when used carelessly. Research on head-up displays in other fields has documented a variety of potential hazards that are relevant to this practice. These include misperception of distance, poorer performance on responses to unexpected objects and obstacles, and cognitive capture [16, 17]. Specifically, an operator who begins to ignore the real world in favor of a head-up display may not notice forward obstacles that are missed by sensors.

One expert cautioned against trying to do too much interpretation of the data. Simple interpretations, like calculating the slope of the terrain is within reason. However, for more complex deductions, it is recommended that the designer should make the data easy to interpret but leave the actual interpretation to the user.

Along these lines, there was discussion of generating virtual representations of the world and robot through collected data and CAD models. With respect to imagery of both the world and vehicle, it was felt that one needs a high degree of accuracy and a good world model. If these are not possible to attain, time consuming and confusing conflicts arise between the virtual imagery and direct data interfaces (e.g., speedometer, roll and tilt gauges). Even if these thresholds are met, "there will be a degree of uncertainty that will be as confusing as it is clarifying."

One expert commented that they once provided a $3 \mathrm{D}$ representation of the robot floating in a window without integrated imagery representing the world (a separate video window was present). "The window was made smaller and smaller, and eventually hidden." It was felt that if CAD models of this sort are not tied to a world model "with extreme accuracy" then they are useless or more damaging than good. 
Finally, the eternal warning regarding care with measurement units was repeated. A suggestion was also issued to make sure interface units match control units.

\section{Control}

There was consistent discussion regarding allowing multiple levels of control. A common practice was to build up from low-level controls (e.g., turn back left wheel $30^{\circ}$ ) to basic teleoperation and then to the desired level of autonomy. Access to all levels of control should be maintained so that an operator can drop down fine control for unusual events. In general, control of the robot should occur at higher levels - independent motor control is clunky and dangerous. Sliding or scalable autonomy was recommended.

The level of autonomy should not be arbitrary; "tailor the degree of autonomy to the circumstance." Furthermore, the user should be able to rapidly identify the robot's level of autonomy - some robots have multiple levels and may shift according to environment or task constraints.

However, total abandonment of human involvement was not recommended. On expert noted that a human in the loop provides adaptability to what would be an otherwise rigid system. This is consistent with a JPL study [18] on humanrobot systems for science oriented mobile robots that documented the benefit of human involvement:

"One could say that current robots are resiliencychallenged, in the sense that generally their skills at responding to anomalies and unexpected events are not fully developed... Humans on the other hand can easily detect samples that are not expected to be in their environment, and can recognize the potential scientific value of their unexpected scientific findings. " [18], p. 3

A key concern was the agreement between operator mental models and robot behavior. "I think the critical factor is not familiarity with the widgets or the GUI but understanding how this mechanism is going to affect the robot." This was also concern regarding the transmission of an accurate control-behavior model to novice or non-expert users. "Most people can learn the interface, it's actually being able to understand what the interface is going to do is the challenge." This was less of a concern for developers and experts as mental model of controls often come from designing and building the robot and frequent operation on challenging terrain.

\section{Command Inputs}

Experts recommended that controls should be flexible to support input from alternative views (e.g., direct joystick for video, waypoint selection for maps). At a general level, the value of tuning controls to the task was echoed by advice to spend time considering how to enhance human-robot communication.

This advice also applies to the selection and design of command inputs. Care should be taken when converting operator commands into robot goals, as the tradeoff between what the robot and operator wants can lead to inefficiency.
Furthermore, most robots utilize deterministic control methods and do not cope well with ambiguous commands or unusual events. Some requests will be obeyed precisely even if the operator only wants approximate behavior.

Scripts and preplanned macro actions (e.g., wall or road following) were reported to be very helpful for semiautonomous systems (e.g., "10 second autonomy"). This functionality is akin to technology already present in the automotive mass market like cruise control and self-parking automation [19].

Experts warned that controlling and navigating with 3D interfaces could be difficult. It is easy to become disoriented and lost when free "flight" motion is permitted.

\section{Status and State}

A general trend was the requirement that operators should be able to rapidly identify the robot's health and motion characteristics. At the basic level are values like direction, speed, tilt, pitch, roll, and health alarms. More arcane values and information that is rarely checked should be relegated to pop-up or secondary screens. Fuel and/or battery charge were not mentioned, but this may be due to the research emphasis of the experts interviewed or that fact that it was considered so essential it was not worth mentioning. It is likely that this will be a critical characteristic for deployments and regular users.

Gauges and state information that changes color or popsup when a threshold crossing occurs are worth considering as a means of reducing display clutter. Pop-up gauges [20] and multi-level attention seeking interfaces for collision warning and steering guidance systems [21, 22] have been successful in automotive applications.

As a general rule, there should be "idiot lights." Impact and state alarms should be grouped together and labeled appropriately and there should be a central error and health summary.

High-level status information regarding subsystems was reported as being useful when diagnosing unusual robot behavior. It was also recommended that secondary information should be grouped by subsystem. This drilldown for details approach is valuable for experts and developers. However, the hierarchy should have clear, logical paths to the desired information.

\section{Recovery}

Autonomous robots always encounter situations where they fail. Experts indicated that it is not possible to design the perfect algorithm so there will always be some breakdown point. As such, robots should be designed to fail into states that are usually safe and recoverable. It is important to understand what this state is for the robot in question. For example, some robots abort into manual control as opposed to a full shutdown. One expert suggested the utilization of "safe modes" that systems can regain calibration and auto-reset if needed.

One expert reported that their team has been devoting more attention to failsafe systems. For mobile robots the 
default response tends to be "hit the brakes" but this sometimes is the wrong thing to do. What may really be needed is to hit the gas, but these situations are subtle and hard to detect.

Humans can spot obvious, yet hard to encode problems. Thus, interfaces should permit rapid situational awareness and overrides. This is not specific to intelligence functions like object classification. It also includes more mundane problems - "In theory it's nice to have automatic cut-offs for things like motor temperature, but the way the sensors are set up it really requires the human to make some judgments." This sort of human assistance requires regular interaction with the robot; a simple problem may go unnoticed if the operator relies on threshold type alerts.

\section{Interface Design}

Perhaps the most unified statement was that most interfaces for fully and semi-autonomous systems are designed by robotics experts for themselves. Most prototype interfaces are not meant to be operated by an untrained user and often include arcane technical information and functionality designed explicitly for debugging. As such, most of these engineering interfaces are "designed badly... look like databases with lots of tabs" but are "OK as long as the people who know how to drive them end up driving them."

The experts recommended human-computer interaction (HCI) methods for interface development and careful testing of interfaces. "In my experience, most robot user interfaces are built with little regard to HCI principles and design techniques." One expert predicted greater need for better interfaces with the migration of robots into non-research tasks and greater use by operators who were not robotics specialists. Similar recommendations have been made as a result of field research on mobile robots [9].

One expert remarked that a good interface takes time to develop and requires incorporation into the team. "If an interface will play a significant role in how the system is used, then interface design needs to be an integral part of development from the very beginning."

There were suggestions that developers strive for a consistent GUI design but avoid locking users into specific information or controls. One expert had gone so far as to develop platform independent interface libraries to allow consistent design across robot systems.

Integration of information was common (e.g., showing camera pointing angles in the compass display). Colorcoding was also reported as useful for indicating confidence (e.g., obstacle detection), important quantities (e.g., brake actuator pressure, engine temperature), and alarm levels.

Experts commented that interface design should account for communications delays. The user should be able to rapidly determine that they are looking at temporally separated information and, if possible, the quantity of separation.

Display choice can have an impact on performance. Daylight readable screens were suggested for outdoor operators. Stereo displays are sometimes helpful but developers should attempt to use shutter glasses over headmounted displays due to fatigue and nausea.

A poor operator environment can render an interface useless by sapping the user's patience and motivation. Related research on urban search and rescue has identified operator environment and sleep deprivation as important factors to consider when designing robot interfaces [9]. While not reported by the experts, long periods of sleep deprivation are also likely in research and prototype development settings.

A general warning was issued to utilize ergonomics when assembling control hardware. On experimenter encountered a system where the steering yoke was in front of a touchscreen. It was necessary to reach around the yoke to access part of the screen.

\section{DISCUSSION}

Classification of expert comments was relatively straightforward in that many of them identified consistent material. This suggests that those producing interfaces for fully and semi-autonomous mobile robots should, at the minimum, ensure that they have addressed the broad categories of: safety, remote awareness, control, command inputs, status and state, recovery, and interface design. Table I summarizes the key lessons within each category.

The suggestion by one expert to integrate interface design early into the development process is especially valid in that the formulation of operator and robot limits and requirements will lead to more cohesive and efficient interaction between humans and robots. The approach of designing the interface after a robot has been developed is suboptimal by default due to the missed opportunity to structure human-robot communication in the early stages of development.

It is important for developers of mobile robots to also realize that it will become increasingly less likely that they will be the sole operators of the robots they fabricate. It is highly likely that the robots will be operated by non-experts and will therefore need methods of accommodating unexpected failures, poorly informed commands, and users who are not well versed in typical robotics terminology or concepts. Furthermore, in the overwhelming majority of cases, monitoring the robotic system's behavior will not be the operator's primary task. In fact, it may not even be a secondary task. As such, there is a growing need for increased research on successful methods for human interaction with autonomous and semi-autonomous mobile robots.

\section{ACKNOWLEDGMENT}

The author would like to thank the interviewed experts for taking time out of their busy schedules and sharing their insight and experience. 
TABLE I. SUMMARY OF LESSONS

\begin{tabular}{|c|c|}
\hline Category & Lessons \\
\hline Safety & $\begin{array}{l}\text { - Safety is paramount } \\
\text { - The vehicle should fail into a safe state for both bystanders and the robot } \\
\text { - Emergency stop controls should be present at both the robot and operator locations } \\
\text { - For multiple operators, consider giving veto power to the operator with a direct line of sight of the robot } \\
\text { - Dangerous actions should not be permitted to occur during calibration and start-up states }\end{array}$ \\
\hline $\begin{array}{l}\text { Remote } \\
\text { Awareness }\end{array}$ & $\begin{array}{l}\text { - The interface should help the user understand the remote environment and maintain situational awareness } \\
\text { - Video and map views are useful, but it is not a requirement for both to be visible at the same time } \\
\text { - Robots with a forward video scene, even at low resolutions, are ripe for mimicking traditional automotive representations } \\
\text { - A dashboard layout on the bottom of the screen to represent key information is useful } \\
\text { - Fused sensor displays (e.g., imagery overlay similar to a head-up display) help but are prone to pitfalls when used carelessly } \\
\text { - Simple interpretations (e.g., calculating the slope of the terrain) are fine, but for more complex deductions, make the data easy to interpret } \\
\text { and leave interpretation to the user } \\
\text { - A high degree of accuracy and a good world model are requirements for generating virtual representations of the world and robot } \\
\text { - CAD models of the robot poorly tied to the world are useless or more damaging than good } \\
\text { - Make sure interface units match control units }\end{array}$ \\
\hline Control & $\begin{array}{l}\text { - Allow multiple levels of control } \\
\text { - General control of the robot should occur at higher levels } \\
\text { Tailor the degree of autonomy to the circumstance } \\
\text { - The user should be able to rapidly identify the robot's level of autonomy } \\
\text { - A human in the loop provides adaptability to what would be an otherwise rigid system } \\
\text { - Agreement between operator mental models and robot behavior is important } \\
\end{array}$ \\
\hline $\begin{array}{l}\text { Command } \\
\text { Inputs }\end{array}$ & $\begin{array}{l}\text { - Controls should be flexible to support input for alternative views } \\
\text { - Seek to enhance human-robot communication } \\
\text { - Care should be taken when converting operator commands into robot goals } \\
\text { - Some requests will be obeyed precisely even if the operator only wants approximate behavior } \\
\text { - Scripts and preplanned macro actions are very helpful for semi-autonomous systems } \\
\text { - Controlling and navigating with 3D interfaces can be difficult }\end{array}$ \\
\hline Status and State & $\begin{array}{l}\text { - Operators should be able to rapidly identify the robot's health and motion characteristics } \\
\text { - Information that is rarely checked should be relegated to pop-up or secondary screens } \\
\text { - Gauges and state information that changes color or pops-up when a threshold is crossed are useful } \\
\text { - There should be idiot lights } \\
\text { - Group and label information appropriately } \\
\text { - Use a central error and health summary } \\
\end{array}$ \\
\hline Recovery & $\begin{array}{l}\text { - Autonomous robots always encounter situations where they fail } \\
\text { - Robots should be designed to fail into states that are usually safe and recoverable } \\
\text { - Consider safe modes for regaining calibration and auto-reseting if needed } \\
\text { - Identify proper default actions } \\
\text { - Humans can spot obvious, yet hard to encode problems } \\
\text { - Permit rapid overrides } \\
\text { - A simple problem may go unnoticed if the operator relies on threshold type alerts }\end{array}$ \\
\hline $\begin{array}{l}\text { Interface } \\
\text { Design }\end{array}$ & $\begin{array}{l}\text { - Most interfaces for fully and semi-autonomous systems are designed by robotics experts for themselves } \\
\text { - Utilize HCI methods and test interfaces } \\
\text { - Expect greater use by operators who were not robotics specialists in applied settings } \\
\text { - Strive for a consistent design but avoid locking users into specific information or controls } \\
\text { - Integrating and color coding information is useful } \\
\text { - Account for communication delays } \\
\text { - Choose display hardware carefully } \\
\text { - Design for potentially substandard operator environments and conditions } \\
\text { - Utilize ergonomics when assembling control hardware }\end{array}$ \\
\hline
\end{tabular}

\section{REFERENCES}

[1] T. W. Fong, C. Thorpe, and C. Baur, "Collaboration, Dialogue, and Human-Robot Interaction," Proceedings of the 10th International Symposium of Robotics Research, Lorne, Victoria, Australia, Springer-Verlag, London, November, 2001.

[2] T. W. Fong, N. Cabrol, C. Thorpe, and C. Baur, "A Personal User Interface for Collaborative Human-Robot Exploration," International Symposium on Artificial Intelligence, Robotics, and Automation in Space, Montréal, Canada, June, 2001.

[3] T. W. Fong, C. Thorpe, and C. Baur, "Robot, Asker of Questions," Robotics and Autonomous Systems, 2003.
[4] P. Padmos and J. van Erp, "Driving with camera view," in A. G. Gale, I. D. Brown, C. M. Haslegrave, and S. P. Taylor, Eds. Vision in Vehicles - V, Amsterdam: Elsevier Science, 1996, pp. 219-228.

[5] A. Oving and J. van Erp, "Driving with a head-slaved camera system," in Proceedings of the Human Factors and Ergonomics Society 45th Annual Meeting, Santa Monica, CA: Human Factors and Ergonomics Society, 2001.

[6] J. Casper and R. Murphy, "Workflow study on human-robot interaction in USAR," in IEEE Conference on Robotics and Automation (ICRA), 2002, pp. 1997-2003.

Accepted for publication at the 2004 IEEE International Conference on Robotics and Automation (ICRA) 
[7] J. van Erp, "Trade-offs between spatial and temporal resolution in driving unmanned ground vehicles," in Proceedings of the Human Factors and Ergonomics Society 42nd Annual Meeting, Santa Monica, CA: Human Factors and Ergonomics Society, 1998, pp.1550-1554.

[8] T. W. Fong and C. Thorpe, "Vehicle Teleoperation Interfaces," Autonomous Robots, vol. 11, no. 1, July, 2001, pp. 09-18.

[9] J. Casper, Human-Robot Interactions During the Robot-Assisted Urban Search and Rescue Response at the World Trade Center, MS Thesis, Computer Science and Engineering, University of South Florida, 2002.

[10] R. Parasuraman, "Human use and abuse of automation," in M. Mouloua and J. Koonce, Eds. Human-Automation Interaction, Mahwah, NJ: Lawrence Erlbaum Associates, 1997, pp. 42-47.

[11] J. D. Lee and N. Moray, "Trust, self-confidence, and operators' adaptation to automation," International Journal of Human-Computer Studies, vol. 40, pp. 153-184, 1994.

[12] A. Steinfeld, and H.-S. Tan, Preliminary Findings for a LaneKeeping and Collision-Warning Driver Interface for Snowplow Operations (PATH Working Paper PWP-99-6), Richmond, CA: University of California, Partners for Advanced Transit and Highways, 1999.

[13] M. Nagamachi, "Ten fatal accidents due to robots in Japan," in W. Karwowski and M. R. Wilhelm, Eds. Ergonomics of Hybrid Automated Systems I, Amsterdam: Elsevier Science Publishers, 1998, pp. 319-396.

[14] V. Verma, "Anecdotes from rover field operations," unpublished, 2000.

[15] J. Holusha, "250,000 Audi cars will be recalled," The New York Times, January 16, 1987, Section A; Page 19, Column 1.

[16] D. Weintraub and M. Ensing, M. Human Factors Issues in Head-Up Display Design: The Book of HUD (CSERIAC State of the Art Report), Wright-Patterson Air Force Base, OH: Crew Systems Ergonomics Information Analysis Center, 1992.

[17] D. Tufano, "Automotive HUDs: The overlooked safety issues," Human Factors, vol. 39, 1997, pp. 303-311.

[18] G. Rodriguez, C. R. Weisbin, and R. W. Easter, A New Method for Human-Robot System Resiliency Evaluation (JPL Publication 02-26), Pasadena, CA: Jet Propulsion Laboratory, California Institute of Technology, 2002.

[19] E. Batista, "Park without dings - or drivers," Wired News, August 25, 2003.

[20] T. B. Sayer and K. Luckscheiter, "A flat panel display for a USMC transport truck," in Proceedings of the Fifth Annual Flat Panel Display Stategic \& Technical Symposium, Ypsilanti, MI: Society for Information Display, Metropolitan Detroit Chapter, 1998.

[21] T. Wilson, W. Butler, D. McGehee, and T. Dingus, T. "IVHS countermeasures for rear-end collisions, driver warning system performance guidelines," in Proceedings of the 1996 Annual Meeting of ITS America, Washington, DC: Intelligent Transportation Society of America, 1996, pp. 949-957.

[22] A. Steinfeld, H.-S. Tan, and B. Bougler "Naturalistic findings for assisted snowplow operations," in Proceedings of the Human Factors and Ergonomics Society 45th Annual Meeting, Santa Monica, CA: Human Factors and Ergonomics Society, 2001. 